\title{
Modelling Contextual Data for Smart Environments. Case Study of a System to Support Mountain Rescuers
}

\author{
Radosław Klimek ${ }^{(\bowtie)}(\mathbb{D}$ \\ AGH University of Science and Technology, \\ al. Mickiewicza 30, 30-059 Krakow, Poland \\ rklimek@agh.edu.pl
}

\begin{abstract}
Context-aware pervasive systems are complex, due to the need to gather detailed environmental information and to perform a variety of context reasoning processes in order to adapt behaviours accordingly. These operations are merged seamlessly. We show the feasibility and vitality of a fully designed system for mountain rescue operations, with various aspects of the contextual processing in middleware, as well as analyse its context life cycle. The system is verified through intensive experiments with a rich set of categorised context data. The contextual processing is shown in different weather scenarios. The service is geared towards software development, converging IoT (Internet of Things) and cloud computing with specific reference to smart application scenarios.
\end{abstract}

Keywords: Streaming sensor data $\cdot$ Modelling contextual information $\cdot$ Middleware $\cdot$ IoT $\cdot$ Rescuing activity

\section{Introduction}

Context-aware systems are analysing complex information which is relevant to a monitored entity and falls into a wide range of data categories $[6,15]$. However, the context understanding presented in the well-known paper by Dey and Abowd [6], seems too general by today's standards, and to be used practically requires categorisation, see paper by Zimmerman et al. [15], introducing a form of interpretation, which allows us to govern the context complexity. In this article, we have categorised the used domain context, which describe the mountain environment. Smart decisions based on various situations and operational scenarios are taken autonomously and pro-actively. System operations are transparent to the sensed entities. Decisions are taken by middleware, which seamlessly binds together all elements.

The first contribution is the categorisation of contextual data for the requirements of mountain environments, especially focusing on supporting mountain rescuers. It is an enabler when reducing complexity, and prioritising activities. Another contribution is a simulation experiment on contextual data processing 
to better understand the nature of defined data, established categories, decision processes, and threats as regular languages. A separate contribution is to holistically verify, thanks to an environment simulator, via a series of experiments, the designed system, which supports mountain rescue operations. Previously, only separate system components were tested, see [9], basing on randomly generated datasets. Presently, the mountain environment simulator produces different datasets. The simulator reflects all the most important aspects of real mountain environments. Five different weather scenarios were proposed and performed. All objectives authenticate system feasibility, credibility and vitality. The system is both an enabler and a provider in increasing understanding of the significance of context-aware decisions, which are based on redundancy, spatial proximity, context transition, context sharing, and other context features. It was designed to help, among other IT engineers, better understand the specifics of context-aware systems.

\section{Related Works}

The basic definition of context was provided by a paper by Dey and Abowd [6] A paper by Zimmermann et al. [15] identified many context taxonomies, user and role, process and task, location and time, amongst others. The first definition seems too general today, since it does not help to govern the complexity of modern software systems. Thus, we introduced the categorisation, which seems the most appropriate for mountain environments. When choosing data for a context, we act in accordance with Crowley's suggestions, see paper [5], that is, only focusing on relevant elements and relationships. A survey by Augusto et al. [3] investigates the notion of context from a historical perspective, as well as showing the relationship between Artificial Intelligence (AI) and Intelligent Environments (IE). A paper by Alegre et al. [1] provides a comprehensive and detailed survey concerning engineering aspects for context-aware applications. It discusses developing methodologies, as well as engineering and conceptualisation for context-aware systems, constituting a solid base for designing their own systems. Hong et al., in a paper [7], state that only a small number of research papers provide development guidelines for context-aware systems, while reducing system complexity can only be achieved by using an appropriate system infrastructure, and context modelling techniques The lack of design techniques is also stated in paper [1].

A paper by Marconi et al. [10] describes a project co-financed by the European Commission, to provide a ground and aerial robotic platform, which supports search and rescue activities in mountain environments. The project does not discuss the fundamental aspects of constant activity monitoring. We are going to show that such an analysis is possible, and can be effective. Our approach is an extension to the aforementioned project, or the beginning of a new one.

This paper is a continuation of [9], where a context-aware and pro-active system to support mountain rescuers was proposed. The current work goes one step 


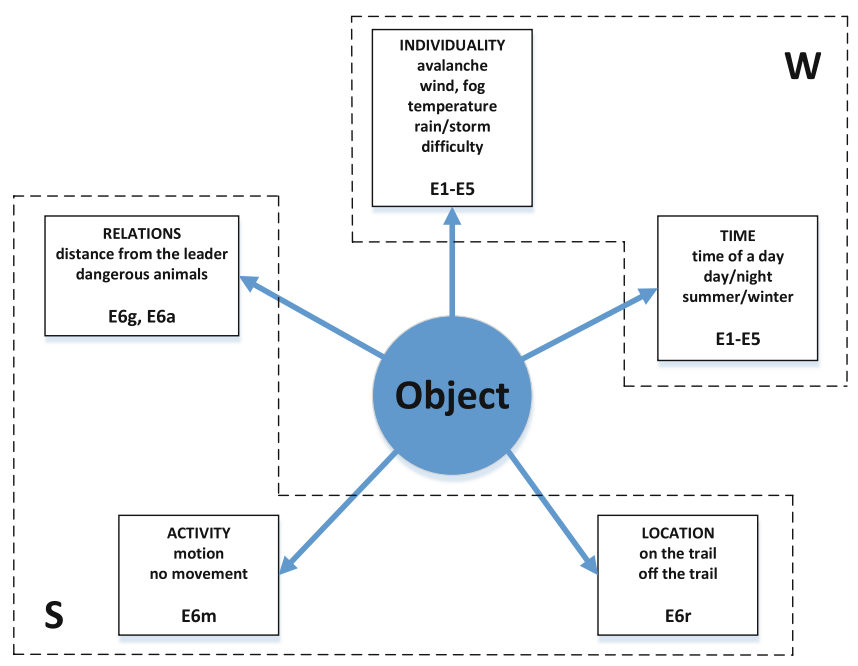

Fig. 1. Assumed context model (with indicated threat levels, see Table 2), see also [9], and division into weather context $W$ and non-weather context $S$

further, because the whole system was built, redesigned as an independent and entire component. In previous works, only message streaming brokers and SAT solvers were tested using randomly generated data. Now, the system was subjected to holistic and comprehensive verification. For this purpose, the mountain environment simulator was created [12]. The obtained contextual data is both important and distinctive for a context-aware system, for example: redundancy, spatial proximity, context transition, context sharing, among others. It is also worth noting that we did not meet many simulators of this type, an exception is a paper by Aronica et al. [2], describing a simulator for rescue operations in marine environments.

\section{Preliminaries}

Information from this section is based on [9, Section III], however assumptions are revised, are clearer, and some understatements and ambiguities are removed. We establish context information categories, see Fig. 1, which influence the monitored object. This context situation is a subject of system predictions.

A division into weather context $W$ (see Table1, threats: E2-E5) and nonweather context $S$ (a dangerous animal on paths, distance from the leader, lack of movement, and on/off the trail, threats: E6a, E6g, E6m, E6r) was implemented. The context data results directly from sensor data and is available for reasoning purposes after the filtration process. Table 2 shows detected threat levels for two context categories, both weather and non-weather categories. 
Table 1. Weather context information, see also [9]

\begin{tabular}{l|l|l|l}
\hline \multicolumn{2}{l|}{ Context } & Labels & Information \\
\hline Avalanche & A1-A5 & $\begin{array}{l}\text { Increasingly difficult } \\
\text { conditions }\end{array}$ \\
\hline \multirow{2}{*}{ Weather conditions } & Wind & W1-W3 & $\begin{array}{l}\text { Increasingly difficult } \\
\text { conditions }\end{array}$ \\
\cline { 2 - 3 } & Fog & $\mathbf{F 1 - F 3}$ & \\
\cline { 2 - 3 } & Temperature & $\mathbf{T 1 - T 3}$ & \\
\cline { 2 - 3 } & Rain, storm & $\mathbf{R 1 - \mathbf { R } 3}$ & \\
\hline Difficulty levels & & $\mathbf{D 1 - D 4}$ & $\begin{array}{l}\text { Increasingly difficult } \\
\text { levels for trails }\end{array}$ \\
\hline
\end{tabular}

Table 2. Increasing threat levels (top row: weather threat symbols, icon colours and danger names; bottom row: non-weather threat symbols and surrounding shapes)

\begin{tabular}{l|l|l|l|l|l}
\hline Weather & E1 & E2 & E3 & E4 & E5 \\
& Green & Yellow & Orange & Red & Black \\
& Low & Medium & Increased & High & Very high \\
\hline Non-weather & & E6g & E6r & E6m & E6a \\
& & Pentagon & Circle & Square & Triangle \\
\hline
\end{tabular}

\section{Contextual Data Processing}

Context creates its context life cycle, that is, the sequence of stages (gathering, modelling, repositoring, reasoning, distribution, and visualisation), which structures processes of contextual pieces of data metamorphosis. Context data goes through particular stages. Starting with data gathering, its pre-processing, or modelling data is located in the repository. After the logical reasoning process, data is distributed in various system locations and visualised. The context data is updated periodically. Figure 2 shows a workflow [11] for the operations of the designed system, however, it is focused on tasks and data flows involving contextual data processing.

Raw data from weather sensors, tourist locations from BTS stations or GPS data, as well as animal geolocation, are placed in Sink. This data is then filtered and modelled, and then tabularised and placed in Repository. After tabularisation, one is able to determine precise weather conditions on specific routes (or their fragments), or assign each tourist to a specific route. Levels of alerts and avalanches are defined manually by mountain rescuers. Repository contains all the tabulated data prepared to make decisions based on logical inference. Recommendations are being prepared for each monitored tourist, according to threat 


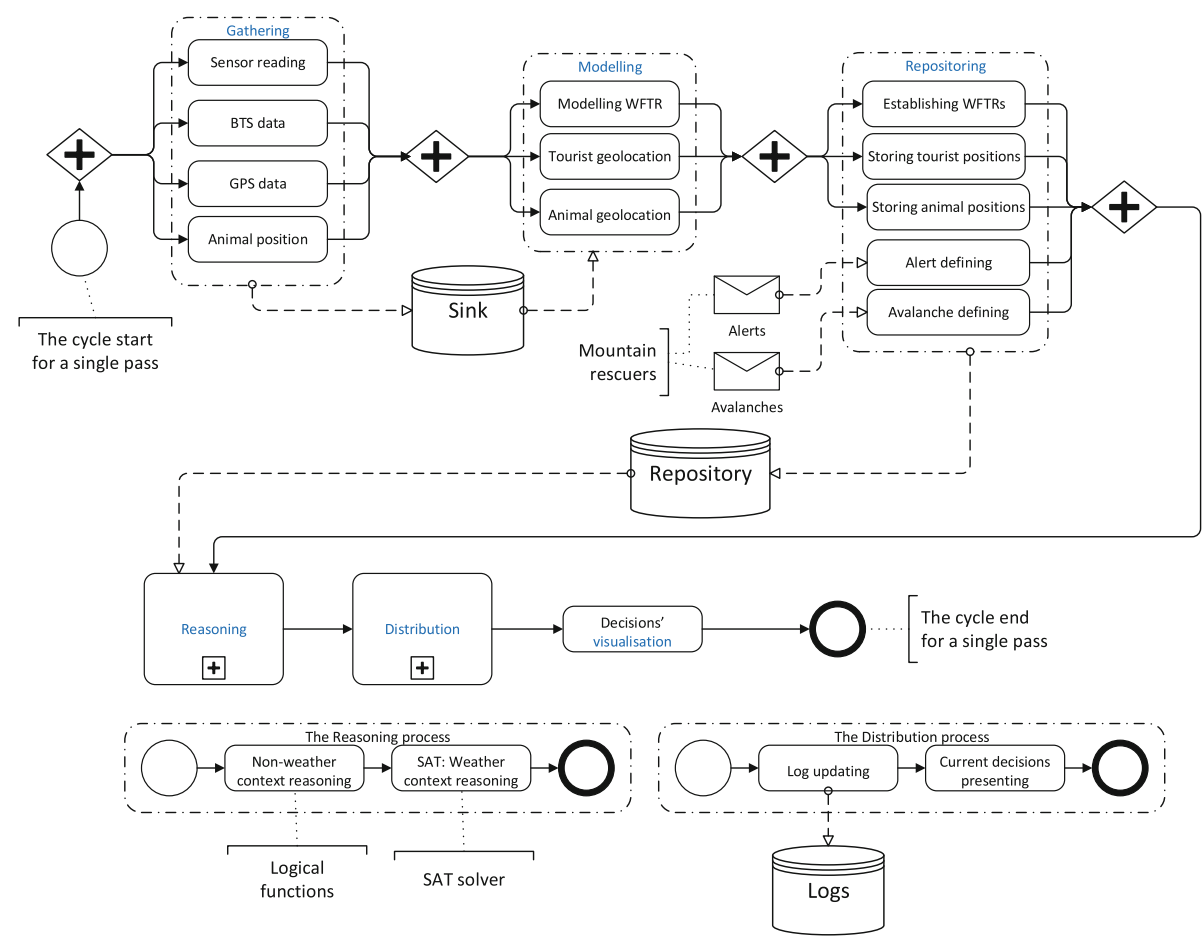

Fig. 2. Workflow for context processing, a single pass: gathering, modelling, repositoring, reasoning, distribution, and visualisation. (WFTR means wind, fog, temperature and rain, as a basic set of weather factors.)

levels, see Table 2. Visualisation occurs on available devices, such as monitors or smartphones, along with the possibility to send text messages.

From a single tourist perspective, threat signals generated by the workflow are described by the regular expression $L_{i} \equiv(E,)^{+}$, where the comma technically separates the single workflow iterations, and $E \equiv N|S| W \mid S \cdot W$, where $N$ means no threat, $S \equiv E 6 a|E 6 g| E 6 m \mid E 6 r$, and $W \equiv E 2|E 3| E 4 \mid E 5$. An example for $L_{i}$ is a finite sentence $N, E 6 a, N, E 6 m E 2, E 3, \ldots$ which ends when the object leaves the monitored area. However, the workflow generates threat warnings for every tourist at the same time, thus $L \equiv L_{1} \cup L_{2} \cup \ldots L_{n}$, where $n$ is the total number of tourists once observed or currently being observed in the monitored area. Thus, every $L_{i}$ and $L$ are regular languages, and are generated by type-3 grammars [8].

We conducted a simple yet interesting simulation regarding the contextual data under consideration, and the results are shown in Fig. 3. The assumptions are as follows: duration time $24 \mathrm{~h}$, sampling every $0.5 \mathrm{~h}$, which gives 48 iterations. Normal distribution for the tourist population, population peak is 200 people at 2:00 pm, and standard deviation $4 \mathrm{~h}$. We examine three time periods: morning 


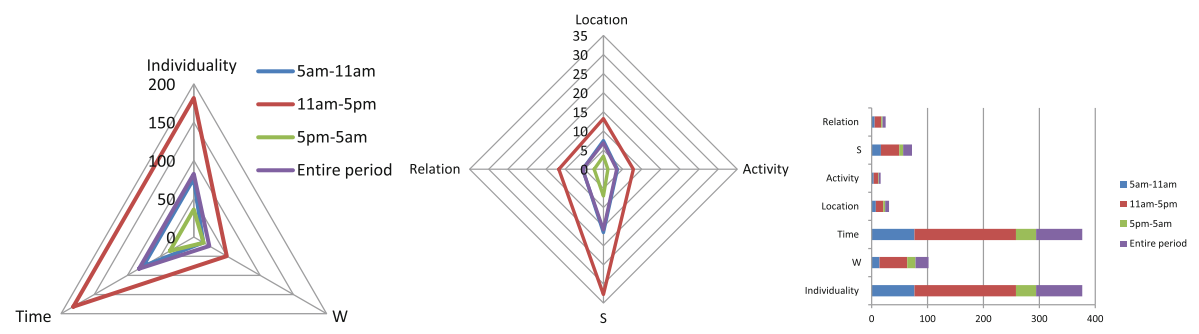

Fig. 3. Simulation of contextual data processing, and two categories $(W, S)$, average values: left - weather context information, middle - non-weather context information, and right - cumulatively all pieces of contextual data. (Blue and violet show similar values and fuse together for the left and middle figures.) (Color figure online)

5:00 am-11:00 am, noon-afternoon 11:00 am-5:00 pm, and evening-night 5 pm5 am. Probability for E2-E5 (considered together) is $20 \%$ for the 1 st time period, increases by $30 \%$ for the 2 nd period, and by $100 \%$ for the 3rd period. Probability for E6a is 5\%, E6g is 5\% (while 30\% tourists are in groups), E6m is 5\%, and E6r is $10 \%$. Threats for both categories are calculated independently. If we have any threat which belongs to $S$, subsequent ones are not calculated, see $[9$, Algorithm 3], which is a result of conditional checking. The use of a particular context data is shown in Fig. 3. Individuality and Time are equally used to calculate the type- $W$ threat. On the other hand, Relation, Activity and Location are used to calculate the type- $S$ threat, however, Relation is used more often (for E6a, E6g). The largest number of threats $W$ occurs with a large number of tourists, see the 11:00 am-5:00 pm period at the right figure. At night, the 5 pm-5 am period, which is the longest one, there are very few tourists, but the threat probability is doubled, which gives a relatively large number of threats. The number of Individuality and Time readings will always be the largest when compared to others, because they concern every tourist. At night, the $5 \mathrm{pm}-5 \mathrm{am}$ period, there are very few tourists and the number of $S$ threats must be small.

\section{Mountain Environment Simulator}

\subsection{Basic Assumptions}

The developed simulator [12] enables generating extensive data, which mirror real mountain conditions within the monitored area and applies to its numerous aspects, for example: weather conditions, tourists' location (trail), walking speed, probability of changing or continuing walking along the same route on trail intersections or the probability of getting lost. Other important aspects of the simulator's work are related to animal migrations. Figure 4 presents screenshots from the simulator system, together with the monitored area. Figure 4 also presents exemplary screenshots of different administrative panels of the simulator. They enable us to influence mountain conditions, and in effect, the datasets generated by the system, by setting particular parameters. 


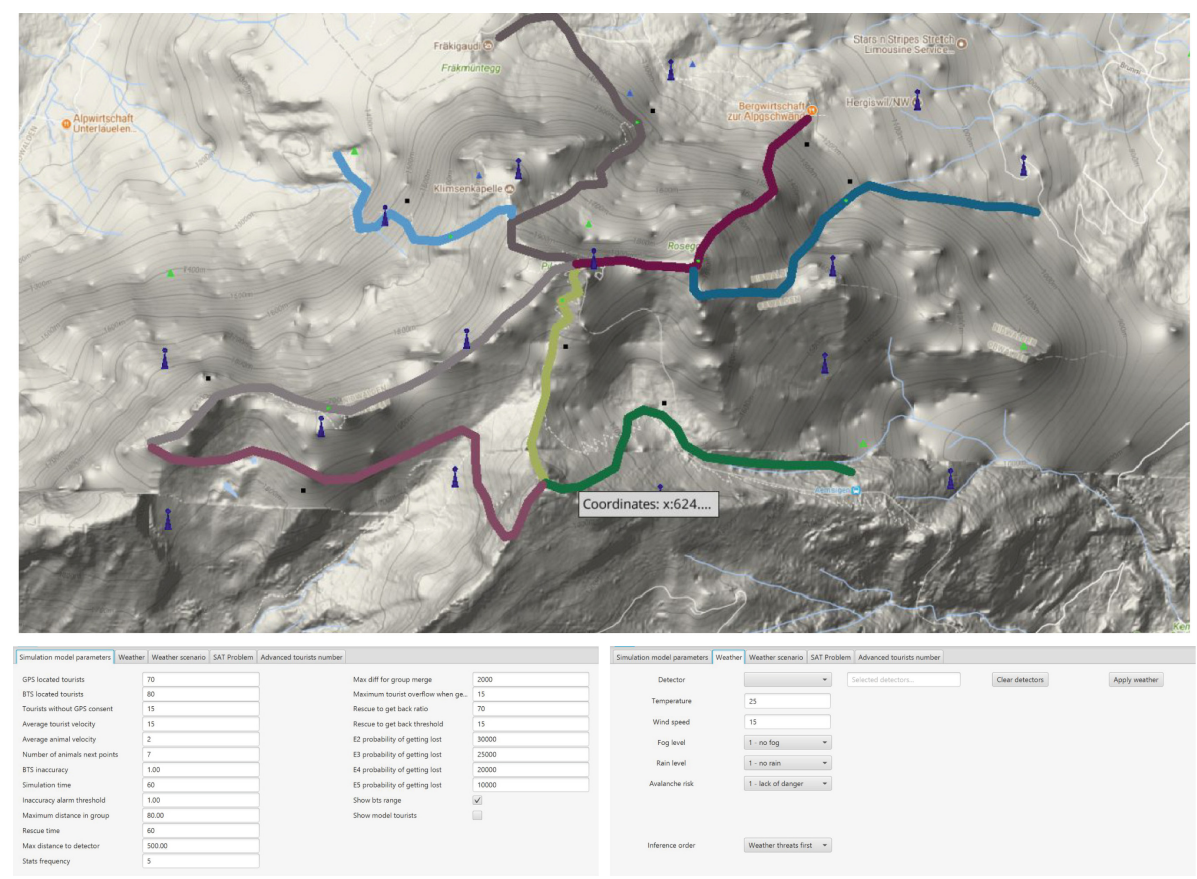

Fig. 4. Simulator screenshots: top - map of the monitoring system before tourist enters (the mountain routes are prepared using QGIS [13] and the base map is from Google Maps), bottom, left - admin panel, the simulation tab [12], bottom, right - admin panel, the weather tab [12]

Let us analyse five different weather conditions, with a separate simulation process being prepared for each case:

1. summer, rather bad weather conditions, but they improve, possible periodical fluctuations;

2. summer, very good weather conditions, but at some point, they significantly worsen (until the end of the simulation process);

3. summer, very good weather conditions, but they worsen for short periods of time;

4. winter, difficult weather conditions, with periodical fluctuations;

5. winter, very difficult weather conditions, but at some point, they significantly improve (until the end of the simulation process).

Each simulation process takes around one hour. and processes 25-30 times faster than real life. In other words: a one-hour simulation is equal to processes which, in a real life mountain scenario, would take approximately thirty hours. Figure 5 presents considered weather scenarios. The total number of weather threats for all tourists within a monitored area was shown. (As a threat we consider all levels from E2 to E5 excluding E1 which describes a normal situation.) 


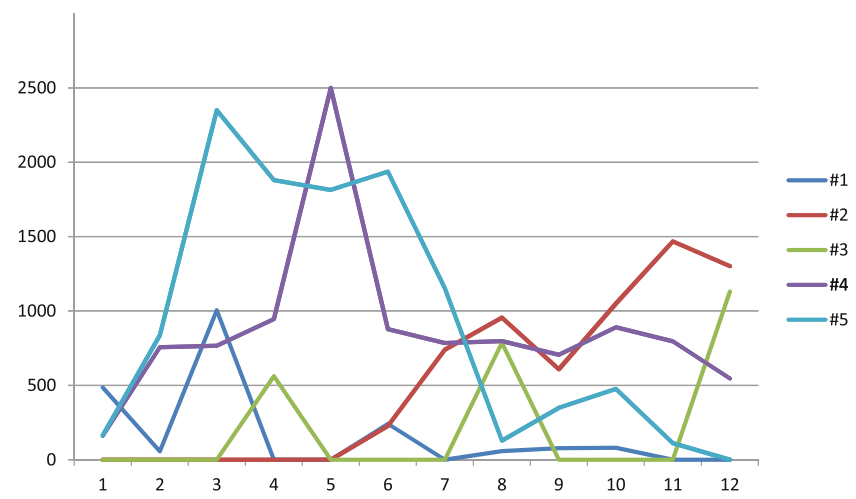

Fig. 5. Total number of detected threats for five different scenarios as a result of weather condition fluctuations. (The entire simulation period is divided into twelve intervals.)

Data is collected at regular intervals, twelve times during one simulation hour, which is equal to collecting data every five minutes. (In general, the frequency of data collection can be established in any other way.) If weather conditions get worse, the frequency of threat detections must increase in relation to objects within the monitored area. We also assume regular division of tourists on routes, as well as the fact that weather changes appear at the same time within the whole monitored area.

Scenario \#1 shows weather fluctuations which stabilise themselves with time. At the beginning, both \#2 and \#3 have excellent weather conditions. In the first example they rapidly worsen, while the second example only experiences some local deviations. Both \#4 and \#5 are related to winter conditions. The first example describes normal weather fluctuations, while the second example is related to very bad weather conditions which gradually improve. Each diagram shows the overall presence of threats. In the case of winter scenarios, there are rather higher threat levels, which range E2 to E5, while during summer there are lower levels from the same range. It is not necessary to show the internal structure of those threats.

\subsection{Simulation Results}

Table 3 shows a general overview of simulation processes, and what happened on the routes within a monitored area. A lot of emphasis has been put on making this image both realistic and reliable. All numbers refer to the total number of events, i.e. events which happened during the hour-long simulation. Only a group of four rows, starting from the third row, concerns the current number of tourists.

"Low BTS location accuracy situations" means situations where the accuracy of the position, determined by the data from BTS stations, is too low. Broadly 
Table 3. Simulation processes' general summary

\begin{tabular}{|c|c|c|c|c|c|}
\hline & $\# 1$ & $\# 2$ & $\# 3$ & $\# 4$ & $\# 5$ \\
\hline Total number of tourists & 3308 & 3344 & 3080 & 3575 & 3298 \\
\hline Tourists who left the area & 3088 & 3123 & 2870 & 3351 & 3060 \\
\hline Current/last number of tourists & 220 & 221 & 210 & 224 & 238 \\
\hline BTS located tourists & 136 & 140 & 129 & 132 & 144 \\
\hline GPS located tourists & 84 & 81 & 81 & 92 & 94 \\
\hline Tourists who denied GPS data & 29 & 33 & 16 & 30 & 26 \\
\hline Animal threats & 628 & 629 & 519 & 417 & 343 \\
\hline Weather threats & 2003 & 6349 & 2478 & 10527 & 11195 \\
\hline Avalanche risk alarms & 20 & 6 & 27 & 18 & 17 \\
\hline "No movement" situations & 84 & 364 & 118 & 836 & 620 \\
\hline "Out of route" situations & 85 & 372 & 122 & 848 & 623 \\
\hline Low BTS location accuracy situations & 1152 & 967 & 637 & 222 & 640 \\
\hline "One weather detector" situations & 3891 & 4027 & 3831 & 4029 & 4176 \\
\hline Tourists who lost their group leader & 149 & 225 & 130 & 470 & 360 \\
\hline
\end{tabular}

speaking, the algorithm works in such a way, see [4], that, by knowing the distance between two stations, it determines two intersection points of the circles which have their centres in the exact location of stations, and radiuses equal to the distance between those stations. Having those two points, we can calculate their distance to the third station. Moreover, knowing the distance between a tourist and the third station, determined from the strength of signal, we can decide which one of the two predetermined points is closer to our result. Discrepancy between the distance determined from the algorithm, and the distance determined from the strength of a signal is treated as an inaccuracy. In the case of the discrepancy being too high, a report is sent to the system, and there is the possibility to send a BTS drone.

The general image presented above is supplemented by the presentation of weather threats which appeared in every simulation process (level E1 describes a normal situation) and the total number of threats on each route, see Table 4 . The data shown proves that the mountain environment was simulated in a realistic way. Numerous simulation aspects concerning weather and non-weather threats were considered.

Redundancy as repetition of information, or inclusion of additional information to improve the quality of processing, occurs in the system when locating objects in the monitored area. The basic way to localise a tourist is to analyse the data from a BTS station. GPS data is obviously much more precise in relation to geolocation, but this data can only be obtained from users who agreed, after entering the monitored area, to such a means of sending data regarding 
Table 4. Total number of recorded weather threats

\begin{tabular}{|l|c|c|c|c|c|}
\hline & $\# 1$ & $\# 2$ & $\# 3$ & $\# 4$ & $\# 5$ \\
\hline \multicolumn{7}{|c|}{ By emergency level } \\
\hline E2 & 1774 & 4438 & 1259 & 3352 & 3301 \\
E3 & 229 & 1797 & 567 & 4669 & 3114 \\
E4 & 0 & 114 & 298 & 1314 & 1696 \\
E5 & 0 & 0 & 354 & 1192 & 3084 \\
\hline
\end{tabular}

\begin{tabular}{|l|c|c|c|c|c|}
\hline & $\# 1$ & $\# 2$ & $\# 3$ & $\# 4$ & $\# 5$ \\
\hline \multicolumn{7}{|l|}{ By routes } \\
\hline Route1 & 742 & 1906 & 682 & 2437 & 2087 \\
Route2 & 0 & 1 & 0 & 1 & 4 \\
Route3 & 55 & 572 & 215 & 1151 & 1180 \\
Route4 & 683 & 1135 & 487 & 2487 & 5053 \\
Route5 & 64 & 283 & 159 & 765 & 1368 \\
Route6 & 32 & 206 & 40 & 523 & 270 \\
Route7 & 49 & 229 & 57 & 457 & 282 \\
Route8 & 378 & 2017 & 838 & 2706 & 951 \\
\hline
\end{tabular}

their location. The process of positioning for each object is possible thanks to the comparison of data from BTS and GPS, if the latter exists. The use of redundancy to resolve the location issues has been shown as a subset of data on the current/last number of tourists in Table 3 .

The benefits of redundancy also apply when considering tourist groups. People can visit a monitored area individually, but can also be organised into registered groups. There are no rules on how large a group can be, but the typical size of a group is 3, 4 or 5 people. (In a small number of cases, there are also two-person groups.) Then, even if one member agreed to send GPS data, it may be helpful to localise other BTS-oriented tourist positions. Table 5 shows the localisation data gathered for a one-hour simulation. It has been proved that the redundancy of information may be successfully used, mainly in relation to tourist locations, which makes the system more effective and precise.

On the other hand, Fig. 6 supplements the above image and presents the volatility of the different kinds of data regarding observed tourists within registered groups. The figure shows data for one simulation scenario, however, the images for the remaining scenarios are very similar. All analysed scenarios prove the credibility of the simulation processes. Fluctuations connected with groups

Table 5. Tourists registered in groups, or redundancy for the group localisation case

\begin{tabular}{l|l|l|l|l|l}
\hline & $\# 1$ & $\# 2$ & $\# 3$ & $\# 4$ & $\# 5$ \\
\hline Total number of tourists in groups & 103 & 82 & 102 & 31 & 102 \\
\hline Number of groups & 24 & 22 & 28 & 9 & 24 \\
\hline Number of BTS located tourists & 90 & 77 & 92 & 27 & 92 \\
\hline Number of GPS located tourists & 13 & 5 & 10 & 4 & 10 \\
\hline Locations improved & 49 & 18 & 33 & 9 & 40 \\
\hline
\end{tabular}




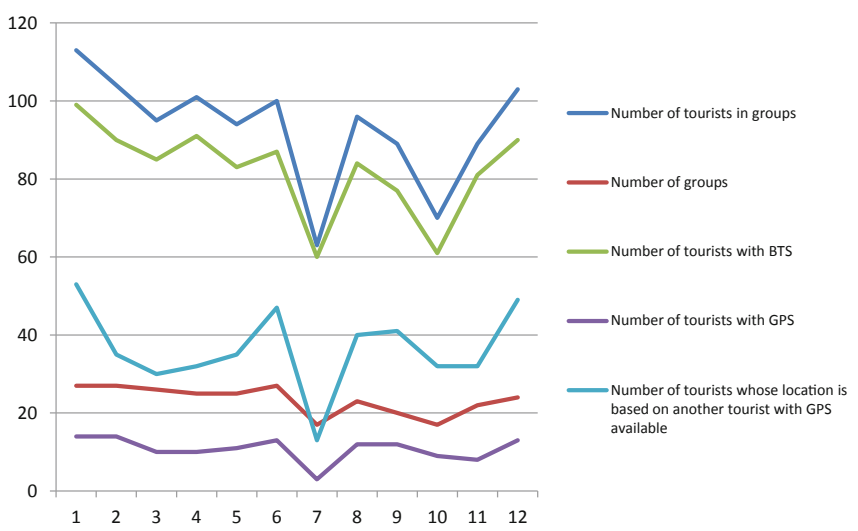

Fig. 6. General view of tourists in groups in the case of simulation \#1. (The entire simulation period is divided into twelve intervals.)

and localisations which follow are a subject of natural volatility. If a tourist remains within a group, the information about the location of other group members, if followed by GPS, is used to prove the BTS location. Thus, it is another example and proof of the reasonable use of data redundancy in the system.

Spatial proximity means nearness or closeness in space. It is an important and compulsory aspect of the analysis of an intelligent system. In our system, it is implemented in relation to weather conditions, precisely speaking, when downloading data which is generated by meteorological stations located on the routes or in their nearest surrounding. If in the close neighbourhood, there are a few stations, but we only require the data from the nearest one, and if they are within a similar distance, we only require the data from the one the object is approaching. During the simulation there were numerous situations where we had to choose between 1-3 stations. Table 6 presents the results concerning meteorological station readings. It needs to be emphasised that although all tourists,

Table 6. Spatial proximity for weather stations

\begin{tabular}{l|l|l|l|l|l}
\hline & $\# 1$ & $\# 2$ & $\# 3$ & $\# 4$ & $\# 5$ \\
\hline Number of analysed tourists & 334 & 365 & 324 & 401 & 382 \\
\hline Total number of events & 1620 & 1723 & 1614 & 1737 & 1772 \\
\hline Including stations: 1 & 427 & 486 & 462 & 474 & 453 \\
\hline Including stations: 2 & 909 & 979 & 885 & 983 & 1033 \\
\hline Including stations: 3 & 284 & 258 & 267 & 280 & 286 \\
\hline Average number of events per tourist & 4.9 & 4.7 & 5.0 & 4.3 & 4.6
\end{tabular}


without exception, were subjected to the same rules of situational evaluation for weather conditions, for the purposes of this particular experiment, that is spatial proximity, we randomly selected a certain representative subset of tourists. In order to illustrate the experiment, every fifth tourist was chosen. Apart from the number of analysed tourists, Table 6 also includes the number of all events for weather data for particular tourists and differentiation of events when 1, 2 or 3 stations were taken into consideration, respectively. The obtained results are representative and credible, and this statement is related not only to an average number of data readings when the tourist remained on the route, but also to the fact that we are mostly dealing with taking two weather stations into consideration. The station closest to the walking direction was chosen as the one which possessed the most useful data to evaluate the tourists' most recent situation.

Context transition means dynamically switching environments which surround and influence object state and behaviour via the pervasive smart system operations that follow. Basically, tourists' context may change regularly, because it is influenced by weather changes, and, indirectly, also by the degree of a particular rule difficulty, time of day, etc. Contextual changes were observed individually, in relation to every single tourist, along its way which may include a set of routes. Table 7 presents the results of research over contextual changes in relation to tourists who finished their hiking and left the monitored area. The data obtained proves the stability of the simulation process, because of the similar values of particular variables. Of course, in real winter condition situations, routes are visited by a fewer number of tourists, however, we did not decide to decrease these values for winter scenarios, since our goal was to verify the simulation processes, alongside each comparable input value for each scenario.

Figure 7 shows the context transitions for each simulation. Every five minutes of the simulation, all important variables are saved, which gives an image of the environment within the system. For that reason, we are able to calculate an average number of context changes per tourist. Also, for this experiment, the obtained results prove the stability and reliability of the simulation processes. In the initial period of each simulation, the average number of changes is lower, which can be explained by having fewer tourists on routes. When the simulation starts, there are no tourists on the routes. That changes as the simulation continues, until it reaches a natural value. (There are numerous well-known national parks which are closed during some designated periods, or as a result of

Table 7. Context transition for tourists who finished their excursions

\begin{tabular}{l|l|l|l|l|l}
\hline & $\# 1$ & $\# 2$ & $\# 3$ & $\# 4$ & $\# 5$ \\
\hline Number of analysed tourists & 3088 & 3123 & 2870 & 3351 & 3060 \\
\hline Average number of transitions per object & 23.26 & 22.34 & 24.50 & 20.36 & 21.58 \\
\hline Minimum/maximum value & $3 / 250$ & $4 / 166$ & $3 / 148$ & $3 / 146$ & $3 / 127$ \\
\hline Standard deviation & 20.82 & 19.41 & 20.61 & 17.11 & 17.67 \\
\hline
\end{tabular}




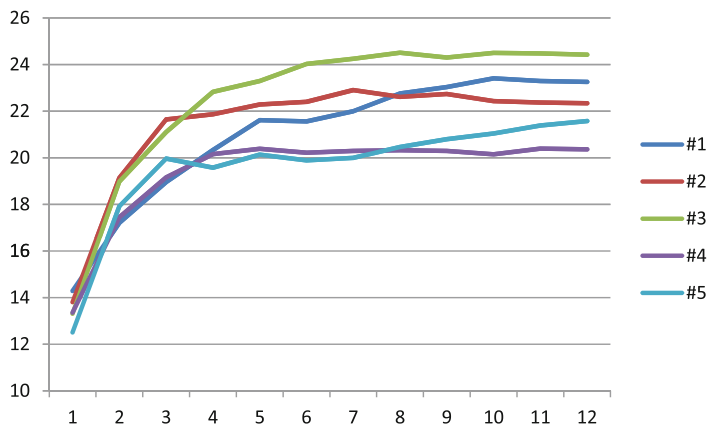

Fig. 7. Context transitions during the simulation, or average number of transitions per object. (The entire simulation period is divided into twelve intervals.)

catastrophes. Opening them and giving access to tourists meets the initial conditions of our simulation scenarios.) All results concerning the context transition prove the experiments success and credibility. In the future, the data may be also subjected to deeper analysis, typical for context-aware and pro-active systems.

Context sharing means overlapping and participation of the information, or knowledge, by different objects. Thus, groups of objects which share context, also share knowledge of how things are perceived within these groups, see also [15]. Figure 8 shows how context sharing is perceived in our system. The results are illustrated in the following way: we have twelve intervals of the simulation process, variables which describe objects are stored as a subject of simulation. Each object is described by using variables to define their context (a touristic route, data from a weather station, difficulty level, BTS/GPS availability). Context sharing was expressed as a percentage in relation to contextual variables. Thus, the most common case is context sharing $25 \%$, with the rest being related to a rather lower number of tourists. $100 \%$ coverage is quite rare. The results obtained are fully natural and prove the credibility of the whole simulation process. The particular percentage groups are pairwise disjointed. The figure is limited to
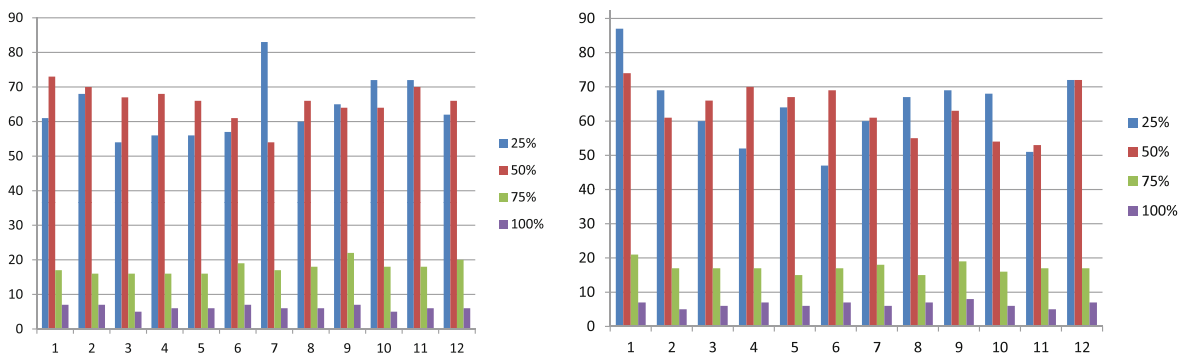

Fig. 8. Context sharing, or number of tourists grouped into four percentages: top summer $(\# 1)$, bottom - winter $(\# 5)$. (The entire simulation period is divided into twelve intervals.) 
winter and summer scenarios. The results were very similar for the remaining seasons. Moreover, context sharing can also be analysed more precisely in relation to particular mountain routes. Again, we conclude that all obtained results are not fundamentally different from our images of naturalness and the credibility of the simulation process.

\section{Conclusions}

We have shown that a sensor-based context-aware system has the ability to sense mountain environments, supporting rescue operations effectively. We have identified contextual elements in accordance with context and system requirements [14]. The number of tourists assumed in all simulation scenarios seems to be quite vast, considering the size of the monitored area. The effectiveness of the proposed solutions were validated by numerous experiments. Some concerns may arise due to the fact that all experiments were carried out on a local host, i.e. both physical phenomena collected by sensors as well as location data together with the monitoring system itself were located in one place. The designed system is also a source of rich analysis focused on contextual data processing. We intend to developed this system to enable defining arbitrary weather scenarios and providing contextual data analysis on demand.

\section{References}

1. Alegre, U., Augusto, J.C., Clark, T.: Engineering context-aware systems and applications. J. Syst. Softw. 117(C), 55-83 (2016). https://doi.org/10.1016/j.jss.2016. 02.010

2. Aronica, S., et al.: An agent-based system for maritime search and rescue operations. In: Omicini, A., Viroli, M. (eds.) Proceedings of the 11th WOA 2010 Workshop, Dagli Oggetti Agli Agenti, CEUR Workshop Proceedings, Rimini, Italy, 5-7 September 2010, vol. 621. CEUR-WS.org (2010)

3. Augusto, J., Aztiria, A., Kramer, D., Alegre, U.: A survey on the evolution of the notion of context-awareness. Appl. Artif. Intell. 31(7-8), 613-642 (2017). https:// doi.org/10.1080/08839514.2018.1428490

4. Calabrese, F., Colonna, M., Lovisolo, P., Parata, D., Ratti, C.: Real-time urban monitoring using cell phones: a case study in Rome. IEEE Trans. Intell. Transp. Syst. 12(1), 141-151 (2011). https://doi.org/10.1109/TITS.2010.2074196

5. Crowley, J.L.: Context driven observation of human activity. In: Aarts, E., Collier, R.W., van Loenen, E., de Ruyter, B. (eds.) EUSAI 2003. LNCS, vol. 2875, pp. 101-118. Springer, Heidelberg (2003). https://doi.org/10.1007/978-3-540-398639_9

6. Dey, A.K., Abowd, G.D.: Towards a better understanding of context and contextawareness. In: Workshop on The What, Who, Where, When, and How of ContextAwareness (CHI 2000) (2000). http://www.cc.gatech.edu/fce/contexttoolkit/

7. Hong, J.Y., Suh, E.H., Kim, S.J.: Context-aware systems: a literature review and classification. Expert Syst. Appl. 36(4), 8509-8522 (2009). https://doi.org/ 10.1016/j.eswa.2008.10.071 
8. Hopcroft, J.E., Motwani, R., Ullman, J.D.: Introduction to Automata Theory, Languages, and Computation. Addison-Wesley (2006)

9. Klimek, R.: Exploration of human activities using message streaming brokers and automated logical reasoning for ambient-assisted services. IEEE Access 6, 2712727155 (2018). https://doi.org/10.1109/ACCESS.2018.2834532

10. Marconi, L., et al.: The SHERPA project: smart collaboration between humans and ground-aerial robots for improving rescuing activities in alpine environments. In: 10th IEEE International Symposium on Safety, Security, and Rescue Robotics (SSRR), College Station, Texas, USA, 5-8 November 2012, pp. 1-4. IEEE (2012)

11. Object Management Group: Business Process Model and Notation (BPMN) version 2.0. Technical report, Object Management Group (2011)

12. Olesek, A.: Simulation of a mountain environment for the analysis of contextual models and data, Engineering diploma thesis, supervisor: Radosław Klimek, AGH University of Science and Technology (2019)

13. QGIS Development Team: Website: QGIS - free and open geographic information system (2018). https://qgis.org/. Accessed 6 Dec 2018

14. Vieira, V., Tedesco, P., Salgado, A.C.: Designing context-sensitive systems: an integrated approach. Expert Syst. Appl. 38(2), 1119-1138 (2011). https://doi.org/ 10.1016/j.eswa.2010.05.006. Intelligent Collaboration and Design

15. Zimmermann, A., Lorenz, A., Oppermann, R.: An operational definition of context. In: Kokinov, B., Richardson, D.C., Roth-Berghofer, T.R., Vieu, L. (eds.) CONTEXT 2007. LNCS (LNAI), vol. 4635, pp. 558-571. Springer, Heidelberg (2007). https://doi.org/10.1007/978-3-540-74255-5_42 